\title{
BMJ Open Role of primary care physician factors on diagnostic testing and referral decisions for symptoms of possible cancer: a systematic review
}

\author{
Victoria Hardy (D) , ${ }^{1}$ Adelaide Yue, ${ }^{1}$ Stephanie Archer (D) , ${ }^{1}$ \\ Samuel William David Merriel (D) , ${ }^{2}$ Matthew Thompson (D) , ${ }^{3}$ Jon Emery (D) , 4 \\ Juliet Usher-Smith (D) , ${ }^{1}$ Fiona M Walter (D) ${ }^{1,5}$
}

To cite: Hardy V, Yue A, Archer S, et al. Role of primary care physician factors on diagnostic testing and referral decisions for symptoms of possible cancer: a systematic review. BMJ Open 2022;12:e053732. doi:10.1136/ bmjopen-2021-053732

- Prepublication history and additional supplemental material for this paper are available online. To view these files, please visit the journal online (http://dx.doi.org/10.1136/ bmjopen-2021-053732)

Received 07 June 2021 Accepted 23 December 2021

Check for updates

(C) Author(s) (or their employer(s)) 2022. Re-use permitted under CC BY-NC. No commercial re-use. See rights and permissions. Published by BMJ.

For numbered affiliations see end of article.

Correspondence to

Victoria Hardy;

veh29@medschl.cam.ac.uk

\section{ABSTRACT}

Background Missed opportunities for diagnosing cancer cause patients harm and have been attributed to suboptimal use of tests and referral pathways in primary care. Primary care physician (PCP) factors have been suggested to affect decisions to investigate cancer, but their influence is poorly understood.

Objective To synthesise evidence evaluating the influence of PCP factors on decisions to investigate symptoms of possible cancer.

Methods We searched MEDLINE, Embase, Scopus, CINAHL and PsycINFO between January 1990 and March 2021 for relevant citations. Studies examining the effect or perceptions and experiences of PCP factors on use of tests and referrals for symptomatic patients with any cancer were included. PCP factors comprised personal characteristics and attributes of physicians in clinical practice.

Data extraction and synthesis Critical appraisal and data extraction were undertaken independently by two authors. Due to study heterogeneity, data could not be statistically pooled. We, therefore, performed a narrative synthesis.

Results 29 studies were included. Most studies were conducted in European countries. A total of 11 PCP factors were identified comprising modifiable and nonmodifiable factors. Clinical judgement of symptoms as suspicious or 'alarm' prompted more investigations than non-alarm symptoms. 'Gut feeling' predicted a subsequent cancer diagnosis and was perceived to facilitate decisions to investigate non-specific symptoms as PCP experience increased. Female PCPs investigated cancer more than male PCPs. The effect of PCP age and years of experience on testing and referral decisions was inconclusive.

Conclusions PCP interpretation of symptoms as higher risk facilitated testing and referral decisions for possible cancer. However, in the absence of 'alarm' symptoms or 'gut feeling', PCPs may not investigate cancer. PCPs require strategies for identifying patients with non-alarm and non-specific symptoms who need testing or referral. PROSPERO registration number CRD420191560515.
Strengths and limitations of this study

- To our knowledge, this is the first study that has examined a range of primary care physician (PCP) factors affecting testing and referral decisions in a cancer context.

- We identified the role PCP factors play in managing 'alarm' symptoms and make recommendations for improving testing and referral decisions for patients with non-alarm and non-specific symptoms.

- We were unable to perform a meta-analysis due to heterogeneity of outcomes among studies.

\section{INTRODUCTION}

Diagnostic error, which encompasses missed, delayed and incorrect diagnosis, is a major patient safety concern in primary care ${ }^{1}$. Cancer is among the most frequently missed diagnoses in this setting internationally. ${ }^{2-4}$ Improving patient safety in primary care has been identified by the World Health Organization (WHO) as a priority. ${ }^{5}$ Achieving a timely cancer diagnosis can be challenging due to low cancer incidence among populations presenting to primary care, ${ }^{6}$ patient comorbidity $^{78}$ and overlapping symptoms between cancers (eg, abdominal symptoms can herald oesophageal, colorectal or renal cancer), ${ }^{9}$ as well as more commonly occurring benign conditions. ${ }^{10}$

Clinical guidelines, such as the National Institute for Health and Care Excellence (NICE) NG12 (2015) guidelines for suspected cancer which are based on patient risk factors and symptoms most predictive of cancer, ${ }^{11}$ are used in several countries to promote timely recognition and referral of symptoms warranting investigation. ${ }^{12}$ Despite reductions in diagnostic delay across multiple cancer sites since implementation of these guidelines in the early 2000s, ${ }^{1314}$ around one-quarter of 
patients with cancer (including those with symptoms of relatively high predictive value; 'alarm' symptoms henceforth) in an analysis of over 17000 cases from the English National Cancer Diagnosis Audit, were deemed to have had avoidable diagnostic delay. ${ }^{15}$ Such patients often have three or more consultations before referral to secondary care for further investigation, ${ }^{16}$ in addition to poor prognosis and experiences of healthcare. ${ }^{17}$

Notwithstanding the inherent difficulty of diagnosing cancer, approximately half of cancer-related diagnostic errors identified from English National Cancer Registries and closed malpractice claims in the USA, have been attributed to PCP delay or failure to recommend an appropriate test or referral. ${ }^{3715}$ Unexplained PCP variation in use of dedicated urgent referral pathways after adjusting for case-mix, ${ }^{18}$ patient and practice factors, ${ }^{19}$ has suggested potential involvement of factors related to the PCP. ${ }^{20}$ However, the PCP factors contributing to decisions to investigate cancer, and the potential benefits and disadvantages such factors may confer to decision making is poorly understood. Therefore, in this systematic review, we aimed to identify and determine the influence of PCP factors on testing and referral decisions for symptoms suggestive of cancer.

\section{METHODS}

This review was conducted in accordance with the Preferred Reporting Items for Systematic Reviews and Meta-Analyses guidelines. ${ }^{21}$

\section{Search strategy}

We searched Medline, Embase, CINAHL, PsycINFO and Scopus for relevant articles from 1 January 1990 to 31 March 2021. We restricted the search from 1990 to coincide with increased public investment for primary care cancer research. ${ }^{22}$ Search queries were developed for Medline (online supplemental material 1) and adjusted according to the conventions of each database. Search terms comprised free text words and Medical Subject Headings informed by key words in titles and abstracts of relevant literature known to the authors, ${ }^{23-25}$ with input from an information specialist.

\section{Eligibility criteria}

Studies were included if they were conducted in a developed country and investigated or described the influence of PCP factors on outcomes of testing and referral decisions during the diagnostic workup of adult patients (aged $\geq 18$ years) with symptoms indicative of any cancer. We defined PCP factors as attributes that have been the object of interest regarding physician performance and competence, specifically focusing on the characteristics and attributes of physicians in clinical practice settings or the community. ${ }^{26}$ PCPs encompassed general practitioners and family physicians who have first patient contact and assume overall responsibility for coordinating patient care. ${ }^{27}$ We did not limit studies by symptom type or thresholds of cancer risk (ie, the positive predictive value of symptom profiles) because we wanted to understand how PCP factors contribute to the management of undifferentiated symptoms that are characteristic of presentations in primary care. As inferring the effect of PCP factors on individual PCP testing behaviour from associations derived in aggregate can be problematic, studies contributing quantitative data were only included if data were reported at the level of the PCP. Systematic reviews were excluded, but where relevant to our review question were used as a source of additional primary articles. We excluded commentaries, letters and editorials. There were no language restrictions.

\section{Study selection}

Citations retrieved by the search were imported into Rayyan QCRI. ${ }^{28}$ Following removal of duplicates, titles and abstracts were screened against eligibility criteria by VH. SWDM independently screened an initial $10 \%$ of titles and abstracts after which the inter-rater reliability (IRR) between reviewers was calculated using Cohen's Kappa coefficient. The resulting coefficient value of 0.66 reflected substantial agreement and exceeded our minimum a priori IRR cut-off of $\geq 0.61,{ }^{29}$ therefore, the remaining titles/abstracts were screened by VH alone. Full texts of potentially relevant studies were independently assessed by VH and AY to determine final inclusion. Reference lists of included studies and systematic reviews were hand-searched for additional articles not retrieved by the database searches. Discrepancies between reviewers were resolved through discussion or adjudicated by SA/FMW when agreement could not be reached.

\section{Data extraction}

Separate data extraction spreadsheets for quantitative and qualitative data were developed and piloted in Excel. Three authors (VH and AY/SA) independently extracted data relating to study characteristics, PCP factors, test and referral type, study results/findings, and cancer using the relevant data extraction spreadsheet. For quantitative studies, VH and AY extracted findings regarding the frequency of PCPs use of diagnostic tests and referrals and subsequent cancer diagnoses, along with odds ratios, hazard or risk ratios, or other statistical measures reported for each PCP factor. For qualitative studies, VH and SA extracted primary data (ie, participant quotations and authors' verbatim summaries and interpretation) with the accompanying themes and subthemes to preserve context for data synthesis. Inconsistencies in data extraction between $\mathrm{VH}$ and $\mathrm{AY} / \mathrm{SA}$ were resolved through discussion.

\section{Critical appraisal}

Quality of included studies was assessed using the Joanna Briggs Institute (JBI) Critical Appraisal Tools as they incorporate appraisal checklists for different study designs. ${ }^{30} \mathrm{VH}$ and $\mathrm{AY} / \mathrm{SA}$ independently rated each criterion of the relevant checklist for each included study, and discrepancies 
were resolved through discussion. As JBI checklists for different study types have a different number of criteria, we summarised study quality using a similar approach taken in a previous systematic review. ${ }^{31}$ The total score for each study was calculated as a percentage and classified as 'low' $(0 \%-45 \%)$, 'fair' $(\geq 46 \%-69 \%)$ or 'high' quality $(\geq 70 \%) \cdot{ }^{31}$ Studies were not excluded on the basis of low quality.

\section{Data analysis and synthesis}

Due to heterogeneity of study outcomes we were unable to statistically pool data. Therefore, we performed a narrative synthesis. We used a convergent segregated approach whereby quantitative and qualitative data were analysed separately before being thematically synthesised. ${ }^{32}$

Quantitative data were initially organised according to the PCP factor label given in included studies. Qualitative data were manually coded for PCPs' perceptions of PCP factors on decisions to investigate cancer that could potentially be grouped into descriptive categories.

Definitions and/or descriptions of PCP factors from quantitative and qualitative studies were compared to determine the similarity of identified constructs. Data for each construct was iteratively grouped and checked by FW/SA until categories of PCP factors became clear. Final labels for each PCP factor were refined following team consensus meetings (table 1).

Quantitative and qualitative findings for each PCP factor were then separately examined. The direction and magnitude of effect (for quantitative data) and PCPs' perceptions (for qualitative data) of PCP factors, were summarised in textual format for every PCP factor. Quantitative and qualitative textual summaries were juxtaposed and subsequently combined into a new descriptive narrative that encapsulated findings from each study. ${ }^{33}$

Finally, PCP factors were organised into over-arching themes according to the extent to which they were deemed to be modifiable or non-modifiable. Modifiable factors were factors considered to be susceptible to individual control or being changed with intervention. Non-modifiable factors were viewed to be outside the purview of individual control and less susceptible to adjustment. Findings for each PCP factor were interpreted in the context of the methodological limitations of each study from the critical appraisal. Only PCP factors for which there were a minimum of two studies were synthesised.

\section{Patient and public involvement}

This systematic review was undertaken as part of the CanTest Collaborative research programme funded by Cancer Research UK. This programme involves close collaboration with a panel of PPI representatives whose views informed the design of this study, data analysis and data interpretation.

\section{RESULTS}

\section{Search results}

The search yielded a total of 7938 studies. After deduplication, titles and abstracts of 4135 studies were screened;
3721 did not meet inclusion criteria and were excluded. We reviewed the full text of 82 studies retrieved from the search, with a further nine identified through handsearching reference lists of included articles. 29 studies (19 quantitative and 10 qualitative) met inclusion criteria and were included in the final synthesis (see figure 1).

\section{Study characteristics}

The included quantitative studies consisted of 13 crosssectional studies ${ }^{2534-45}$ and two experimental studies, ${ }^{2346}$ of which 11 used vignette-based methods; ${ }^{23}$ 25 35-39 42, 44-46 three were prospective cohort studies, ${ }^{47-49}$ and one was a retrospective cohort study (online supplemental material 2). ${ }^{49}$ Qualitative data came from five interview studies, ${ }^{50-54}$ two focus group studies, ${ }^{55}{ }^{56}$ two cross-sectional survey studies $^{57} 58$ and one retrospective cohort of free-text primary care consultation data. ${ }^{59}$ Of these 29 included studies, 11 were conducted in the UK, ${ }^{2325343743445051545557}$ six in Denmark, ${ }^{4041} 45476061$ two across 20 countries within the European Union, ${ }^{39} 58$ two each in Australia, ${ }^{35}{ }^{46}$ the USA, ${ }^{36} 38$ and Norway, ${ }^{49} 53$ and one each in the Netherlands, ${ }^{59}$ Sweden, ${ }^{52}$ Spain, ${ }^{56}$ and across Australia and the UK. $^{42}$ Together, studies reflected a total of 10300 PCPs (8000 from quantitative studies, 2300 from qualitative studies) and testing and referral decisions for 15100 patients.

The most common diagnostic action evaluated was urgent referral ${ }^{3741-435154555760}$ or direct referral to diagnostic testing centres, ${ }^{34} 404550$ imaging tests, ${ }^{48}$ or other specialty services. ${ }^{35} 46$ Additional investigations included use of appropriate investigations for the cancer, ${ }^{23} 25363839$ any diagnostic action, ${ }^{47} 4950525358$ or referral in general. ${ }^{56}$ Testing and referral decisions were mainly made in the context of symptoms generally suggestive of the target cancer(s). ${ }^{35} 3638414748558-60$ The remaining studies examined decision making for symptoms at high-, ${ }^{46}{ }^{49}$ low-,${ }^{25} 34394050$ and mixed-risk of cancer (based on the positive predictive value of symptoms in national guidelines), ${ }^{23} 374244$ or did not provide details of symptomatic context. ${ }^{43} 45$ 51-54 5657 Cancers studied were colorectal, $^{36} 37424352545559$ lung, ${ }^{2344465561}$ ovarian, ${ }^{3860}$ a mixture of cancers, ${ }^{253539-4158}$ and any cancer. ${ }^{3445475051535657}$

\section{Quality of included studies}

Twelvestudieswereassessedashighquality, $23253436373945-47495053$ 11 were fair quality, 3538404144485152555960 and six were low quality (online supplemental material 2). ${ }^{42} 435456-58$

The main quality issues for quantitative studies related to poor reporting of sample characteristics, ${ }^{4014347-4960}$ failure to identify or adjust for confounding, ${ }^{38} 424349$ and hindsight bias due to knowledge of the diagnosis at the point of assessment of PCP factors. ${ }^{40} 4149$ There were also concerns about several studies using vignettes which were not adequately realistic of patient cases seen in clinical practice and lacked evidence of validation. ${ }^{25} 35374657$ Qualitative studies were limited by insufficient evidence of researcher reflexivity and positionality, ${ }^{50-555759}$ suboptimal presentation of participants' 


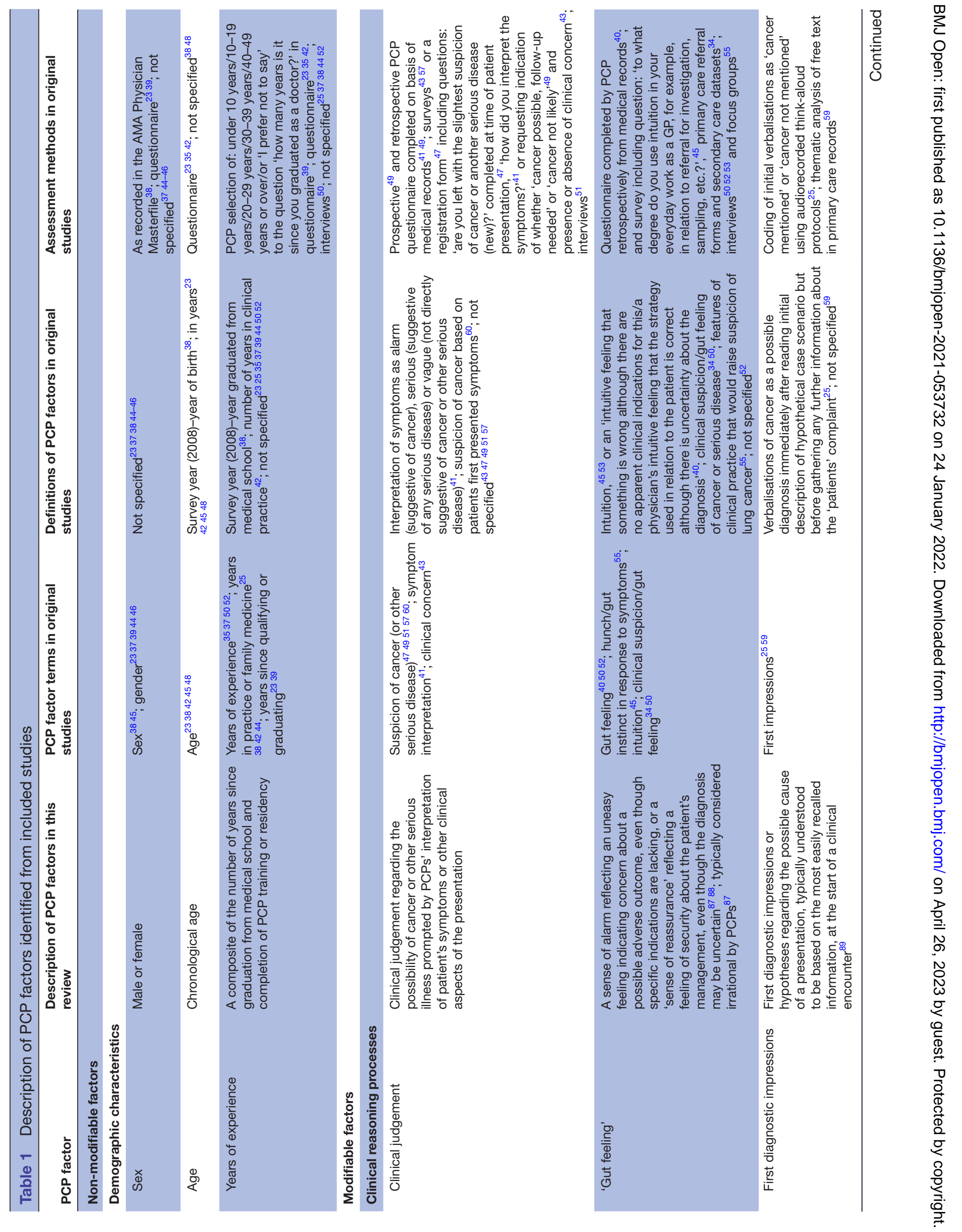




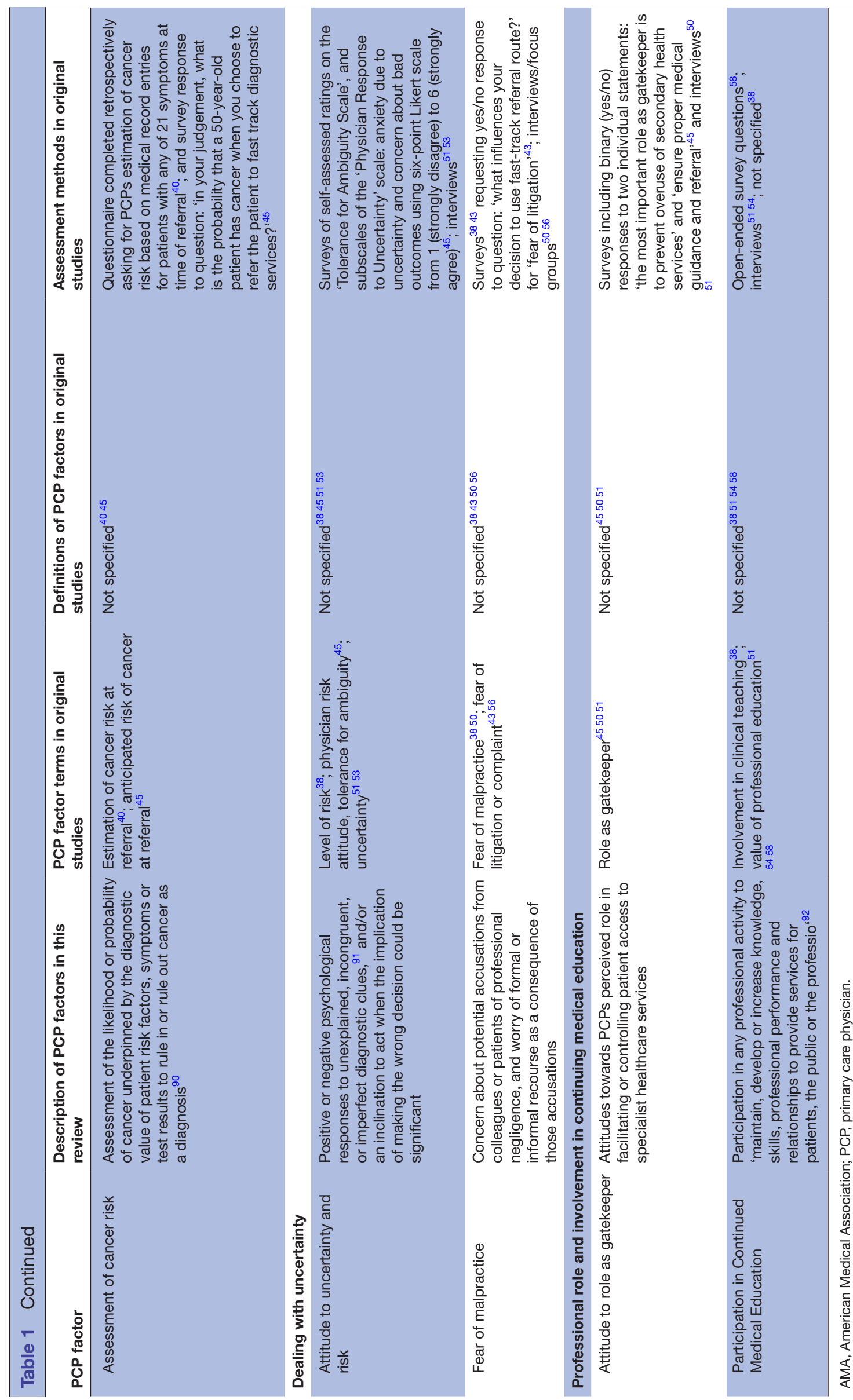




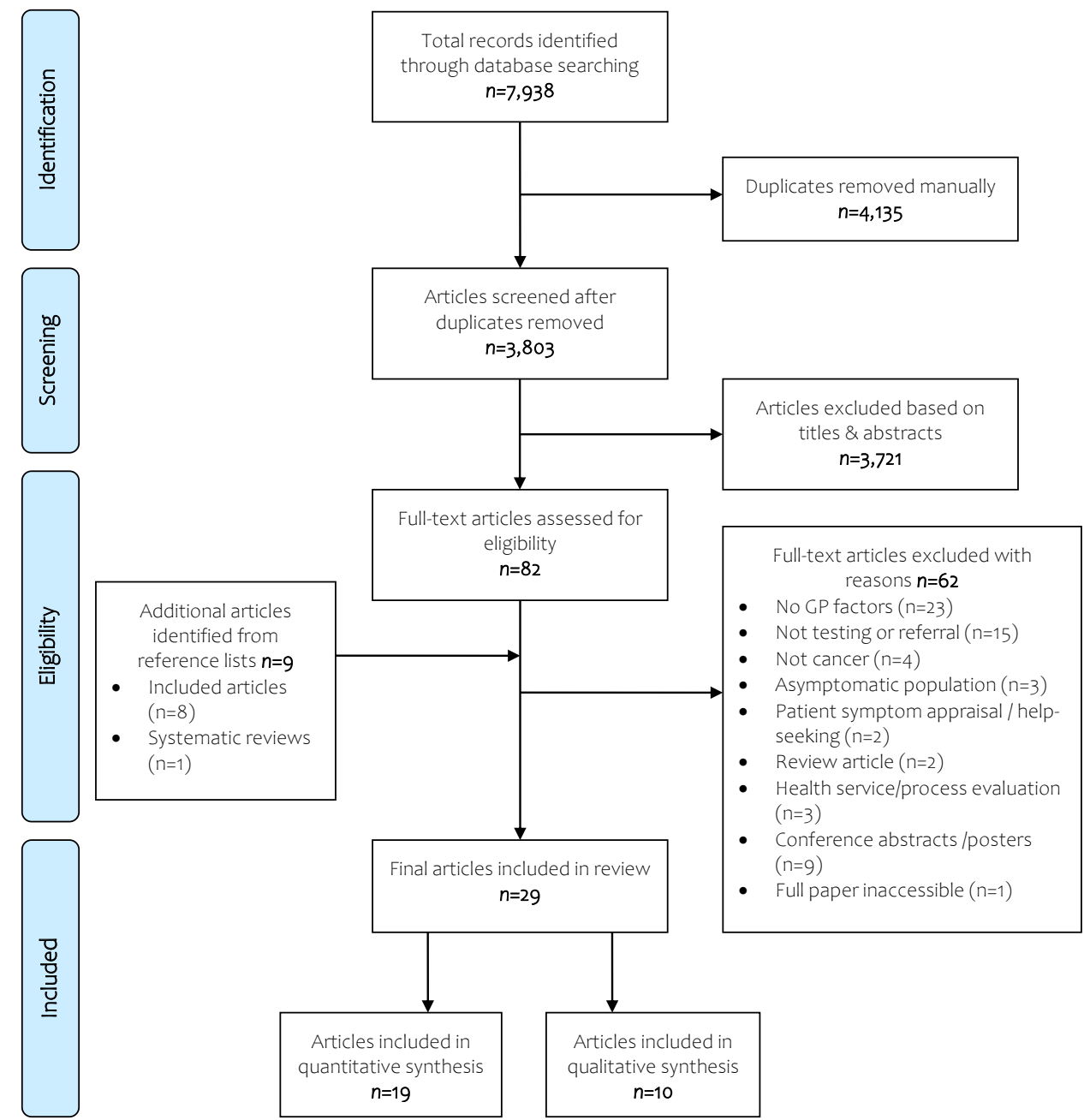

Figure 1 PRISMA flow diagram. PRISMA, Preferred Reporting Items for Systematic Reviews and Meta-Analyses.

voices $^{55} 5659$ and failure to indicate whether ethical permissions were obtained or waived. ${ }^{56}$

Recall and social desirability bias was also a concern across study types where assessment of the exposure by PCPs was examined retrospectively, ${ }^{40} 4149$ or based on PCPs reflections of their clinical practice. ${ }^{50-54}$

\section{PCP factors}

A total of 11 PCP factors were identified (table 1). Nonmodifiable PCP factors comprised demographic characteristics. Modifiable PCP factors included clinical reasoning processes, dealing with uncertainty, and professional role and involvement in continuing medical education. The evidence for these 11 PCP factors is summarised below (quantitative and qualitative findings for each factor are presented separately in online supplemental materials 3 and 4, respectively).

\section{Non-modifiable factors}

Demographic characteristics

$\operatorname{Sex}(n=7)$

Most studies suggested that female PCPs more often investigated possible cancer compared with male PCPs, ${ }^{37-39} 4648$ though findingswere predominantly descriptive. Beingfemale was associated with increased odds of urgent specialist review for patients with a lung nodule on CT (OR 1.87,95\% CI 1.36 to $2.56, p \leq 0.001)^{46}$ and referral of symptoms suspicious of colorectal cancer. ${ }^{37}$ Compared with male PCPs, females more frequently recommended appropriate investigations for ovarian cancer $^{38}$ and symptoms of any possible cancer, ${ }^{39}$ but these differences were not statistically significant. There was also no observable trend between PCP sex and use of investigations for lung cancer, ${ }^{23} 48$ nor was there an association with estimation of cancer risk at urgent referral. ${ }^{45}$

\section{Age $(n=5)$}

The effect of age on decisions to investigate possible cancer was inconclusive. In two studies in Australia/UK and the USA, younger PCPs (aged 30-39 years) were most likely to recommend specialist referral for colorectal cancer $^{42}$ and request appropriate investigations for ovarian cancer. ${ }^{38}$ However, in another study conducted in the UK, younger PCPs overall used relevant investigations for lung cancer less frequently than PCPs older than 45 years. ${ }^{23}$ Odds of urgent referral increased with age in one study. PCPs 60-69 years were significantly more likely 
to recommend urgent referral (OR 15.4,95\% CI 4.4 to $53.8, \mathrm{p} \leq 0.001)$ than those $30-39$ years (OR 2.8,95\% CI 1.5 to $5.2, \mathrm{p} \leq 0.001) .{ }^{42}$ PCPs 60 years and above tended to estimate cancer risk at referral to be higher than PCPs younger than 45 years, though this finding was nonsignificant. ${ }^{45}$ There was also no difference in mean age at referral between PCPs using and not using direct CT referral for lung cancer. ${ }^{61}$

\section{Years of experience $(n=10)$}

Evidence regarding the influence of years of experience was also mixed. Compared with those less experienced, PCPs with upwards of 10 years of experience were more likely to make a specialist referral for symptoms of ovarian ${ }^{35}$ and colorectal cancer, ${ }^{42}$ and initiate diagnostic action in general. ${ }^{39}$ PCPs trusted their clinical judgement with increasing experience and became more willing to investigate when they were suspicious of cancer, regardless of colleagues opinions of their decisions. ${ }^{50} 52$ However, one study found PCPs with less than 6years of experience were most able to differentiate presentations at risk thresholds of $3 \%$ or more requiring urgent referral compared with PCPs with 18-36 years of experience. ${ }^{44}$ A further three studies found no observable influence of years of experience on decisions to test and refer for possible cancer. ${ }^{23} 2538$

\section{Modifiable factors \\ Clinical reasoning processes \\ Clinical judgement ( $n=7)$}

Clinical judgement of symptoms as suspicious or alarming consistently led to more investigations across multiple cancer sites. ${ }^{41} 434749515760$ When PCPs were suspicious of cancer they were more likely to recommend imaging investigations (OR $3.95,95 \%$ CI 2.80 to 5.57) or make a referral (OR 2.56,95\% CI 2.22 to 2.96) compared to when symptoms were interpreted as not suspicious. ${ }^{47}$ Symptoms that did not provoke suspicion ${ }^{60}$ or were interpreted as non-alarm ${ }^{41}$ were less likely to be urgently referred. PCPs had difficulty discerning the appropriateness of using urgent referral pathways when patients symptoms did not clearly match referral criteria which was perceived not to accommodate individual clinical judgement. ${ }^{57}$

\section{'Gut feeling' ( $n=7)$}

The presence of 'gut feeling' did not lead to increased referral rates, but doubled the odds of a cancer diagnosis within 6-months when PCPs decided to act on this feeling. ${ }^{340}$ 'Gut feeling' was perceived to facilitate management of symptoms that were not definitive ${ }^{55} 57$ and become more accurate with experience. ${ }^{50} 55$ Justifying referral of patients with concerning symptoms not meeting referral criteria on the basis of 'gut feeling' was challenging as it was perceived to not be sufficient indication for referral among hospital specialists. ${ }^{5053}{ }^{57} \mathrm{PCPs}$ ' assessment of cancer risk at referral was not influenced by 'gut feeling'. ${ }^{45}$
First diagnostic impressions $(n=2)$

In one vignette study, PCPs whose first diagnostic impressions were of possible cancer were more likely to recommend investigation than PCPs whose initial impressions did not include cancer (OR $1.98,95 \%$ CI 1.10 to 3.57 , $\mathrm{p} \leq 0.01) .{ }^{25}$ Further, odds of a cancer diagnosis doubled when PCPs verbalised cancer as a possibility after reading the reason for the patients presentation compared with when they did not mention cancer. ${ }^{25}$ Failure of PCPs to reconsider initial diagnostic hypotheses could herald suboptimal testing strategies and delayed colorectal cancer diagnosis. False reassurance from positive response to medication, intermittent symptoms, misleading test results, and comorbidity were reported to contribute to failure of PCPs to consider alternative diagnostic hypotheses. ${ }^{59}$

\section{Assessment of cancer risk $(n=2)$}

The relationship between PCPs' assessment of cancer risk and use of investigations was unclear. In a prospective cross-sectional study, higher estimation of cancer risk did not result in higher referral rates but did lead to a concomitant increase in the likelihood of a cancer diagnosis. ${ }^{40}$ Findings from a Danish vignette survey suggested that PCPs overestimate cancer risk as approximately onethird of PCPs' anticipated cancer risk at referral for a hypothetical patient to exceed $50 \%{ }^{45}$

\section{Dealing with uncertainty}

Attitude to uncertainty and risk $(n=4)$

Findings for this PCP factor were inconsistent. Survey responses on the Tolerance for Uncertainty scale suggested that PCPs most tolerant of uncertainty were the least confident in their assessment about the possibility of an underlying cancer at urgent referral compared to PCPs with the lowest tolerance for uncertainty. ${ }^{45}$ PCP responses to the Physician Risk Attitude scale and 'levels of anxiety due to uncertainty' and 'concern about bad outcome' domains in the Physician Reaction to Uncertainty scale, was not associated with assessment of cancer risk at referral. ${ }^{45}$ Another study found that decisions to test for ovarian cancer were not affected by PCPs attitude to risk. ${ }^{38}$ Diagnostic uncertainty was viewed as integral to clinical practice, ${ }^{53}$ but could lead younger doctors who were less comfortable with uncertainty to investigate unnecessarily. ${ }^{5153}$

\section{Fear of malpractice $(n=4)$}

The potential influence of fear of malpractice diverged by study type. In two quantitative studies, PCPs concerns about malpractice did not influence selection of tests for ovarian cancer, ${ }^{38}$ nor did PCPs consider fear of malpractice to influence their decisions to use urgent referral pathways. ${ }^{43}$ In contrast, qualitative studies suggested that previous experience of complaints from patients or colleagues for suboptimal testing decisions could affect PCPs future diagnostic approach. ${ }^{56}$ Defensive testing was considered the only strategy available to PCPs concerned 
about litigation for reassuring patients and protecting themselves medicolegally. ${ }^{50}$

\section{Professional role and involvement in continuing medical education}

Attitude to role as gatekeeper $(n=3)$

Evidence for this PCP factor was predominantly descriptive. PCPs believed their role was to act in the best interests of the patient, advocate for patients healthcare needs with hospital specialists, ${ }^{51}$ and make appropriate management decisions. ${ }^{45}$ PCPs' attitudes were not associated with their assessment of cancer risk at referral. ${ }^{45}$ In their role as gatekeeper, PCPs had mixed encounters with hospital specialists when trying to make a referral on the basis of 'gut feeling'. They described productive dialogue with specialists via telephone ahead of a referral, but were reluctant to write 'gut feeling' into the referral letter. ${ }^{50}$

\section{Participation in continued medical education $(\mathrm{n}=4)$}

In one US vignette study, PCPs involved in clinical teaching were 1.04-fold more likely to use relevant tests for ovarian cancer compared with PCPs not participating in educational activities. ${ }^{38}$ Across three interview studies, PCPs wanted more educational opportunities to better differentiate symptoms that could be due to cancer. ${ }^{515458}$ Frequent training on the latest evidence regarding the predictive value of symptoms for cancer was viewed as important $t^{51}$ as lack of clarity about when to suspect cancer was believed to have contributed to incorrect non-referral of patients in their practice. ${ }^{548}$

\section{DISCUSSION}

\section{Summary of principal findings}

We identified a number of non-modifiable and modifiable PCP factors potentially influencing testing and referral decisions for cancer. Of the PCP factors deemed modifiable, we found most evidence for 'clinical judgement' and 'gut feeling'. PCP judgement of symptoms as suspicious or 'alarm' led to more investigations for possible cancer than symptoms judged to be non-alarm. The presence of 'gut feeling' at referral increased the likelihood of a subsequent cancer diagnosis. PCPs relied on 'gut feeling' to guide decisions to investigate non-specific symptoms. Patients' symptoms could not always be easily reconciled with clinical guidelines, making it difficult for PCPs to determine the appropriateness of referral. Clinical guidelines offered limited scope for PCPs to act on 'gut feeling' or clinical judgement when symptoms did not fit referral criteria. Female PCPs tended to investigate cancer more than male PCPs. The effect of years of experience was inconclusive, but more experience was perceived by PCPs to improve the reliability of 'gut feeling'. The evidence for the remaining PCP factors was insufficient to derive clear conclusions.

\section{Strengths and limitations}

To date, research evaluating possible reasons for inconsistencies in PCPs use of suspected cancer referral pathways ${ }^{20}$ have primarily focused on the influence of patient, ${ }^{62}$ practice $^{1663}$ and health-system factors. ${ }^{6465}$ By summarising quantitative and qualitative evidence for PCP factors, this review builds on existing knowledge regarding the range of factors affecting PCPs decisions to investigate cancer and highlights PCPs' perspectives regarding the potential value of those factors in clinical practice. Our search strategy covered a variety of terms for PCP factors increasing the likelihood that all relevant studies were identified. We used a transparent approach to derive PCP factors which could be useful for developing uniform definitions of these factors that can be applied in future primary care cancer research.

However, this study does have some limitations. While we attempted to synthesise data for PCP factors representing similar constructs, heterogeneity in labelling of PCP factors between studies, which were seldom defined, may have affected construct validity. For example, studies reporting clinical suspicion and 'gut feeling' did not always adequately define these terms or describe how they were assessed, making it difficult to determine which construct was actually measured. Additionally, we did not limit evaluation to PCP factors assessed as a primary or secondary outcome, so findings for years of experience, age and sex, in particular, should be interpreted with some caution. These factors were typically examined ad hoc and may not be sufficiently powered to detect a true relationship with testing and referral decisions. While clinical decision making occurs in the context of the patient agenda and wider health system, ${ }^{66} 67$ a number of included studies did not adjust analyses for confounding from patient or health system factors, potentially obscuring the true relationship of PCP factors (notably those relating to PCP demographic characteristics, 'clinical judgement', attitude to uncertainty/risk and fear of malpractice) with testing and referral decisions.

Dichotomising factors as modifiable or non-modifiable may obfuscate the complex and dynamic mechanisms through which PCP factors influence decision making, and the extent to which these factors may be modifiable in practice. For example, PCPs' interpretation of symptoms as 'alarm' or non-serious (ie, 'clinical judgement') may be due to variations in PCP knowledge of referral criteria for suspected cancer or application of cognitive shortcuts (eg, availability heuristic, overconfidence). Although there is scope for these respective components to be improved through interventions such as clinical updates or metacognitive practices that highlight the impact of errors in cognition on patient care ${ }^{68-70}$ knowledge of clinical guidelines may be more susceptible to adjustment than PCPs use of heuristics which is influenced by personality traits that are more difficult to modify. 'G1 'Gut feeling' is associated with empathy ${ }^{72}$ and underpinned by clinical knowledge and experience. ${ }^{73}$ Thus, the ability of PCPs to access and leverage 'gut feeling' in interventions for 
enhancing empathy ${ }^{75}$ may be more instinctive to some PCPs than others. Though years of experience is not modifiable, clinical experience may be more important and mutable through simulated diagnostic experiences that increase exposure to presentations that could be due to cancer. ${ }^{76}$

The overall conclusiveness of our findings is also limited by methodological weaknesses of studies using retrospective (eg, interview, medical record review) methods that are susceptible to recall, social desirability and hindsight bias.

\section{Comparison with existing literature}

That PCPs were less likely to investigate symptoms judged to be 'non-alarm' comports with existing literature which reports longer diagnostic intervals for lower risk symptoms not meeting urgent referral criteria across cancer sites ${ }^{77}$ and non-investigation or delayed investigation of patients with gynaecological cancers presenting to primary care with non-specific symptoms. ${ }^{78} \mathrm{~A}$ recent systematic review and meta-analysis examining the influence of 'gut feeling' in the diagnosis of cancer similarly found 'gut feeling' to be highly predictive of cancer (OR 4.24,95\% CI 2.26 to 7.94) and linked with PCP experience. ${ }^{74}$ In the same review 'gut feeling' also led to more referrals ${ }^{74}$ which was not supported by our findings. This inconsistency may be due (in part) to differing interpretations of PCP factors constituting 'clinical judgement' and 'gut feeling' (for example, we deemed clinical suspicion in two similarly included studies ${ }^{47}$ to reflect 'clinical judgement' rather than 'gut feeling'). Qualitative studies of cancer patients symptom appraisal suggest patients use vocabulary when communicating their symptoms that differs from biomedical terms familiar to PCPs,${ }^{79-81}$ which may explicate the difficulties PCPs experienced reconciling patients' symptoms with referral criteria. A crosssectional observational study of English practices found that practices with majority male PCPs were less likely to urgently refer for suspected cancer, ${ }^{82}$ which accords with our finding that female PCPs were more inclined to investigate patients with symptoms of possible cancer.

\section{Unanswered questions and future research}

Future research in this area would benefit from standardised definitions and reporting of PCP factors. In the UK, the amalgam 'clinical suspicion/gut feeling' is indicated for expedited referral to non-specific symptom pathways, and NICE NG12 (2015) ${ }^{11}$ urgent referral criteria recommends clinical judgement is used when making appropriate testing decisions. ${ }^{11}{ }^{34}$ However, the extent to which 'clinical suspicion' or 'clinical judgement' (both assessed as 'clinical judgement' in this review) and 'gut feeling' reflect distinct or overlapping constructs is unclear. Research is needed that elucidates how PCPs appraise symptoms as suspicious or non-suspicious to determine patient eligibility for referral. Consideration should be given to the subsequent impact on decisions to investigate possible cancer when symptoms are not described in biomedical terms. PCP factors are multidimensional and comprise contributory components that may be more modifiable than others. Disentangling the potential involvement of such components for 'clinical judgement' and 'gut feeling' could foster more understanding of these constructs and facilitate identification of factors to target in future interventions. Testing and referral decisions may be driven by aspects of PCP gender as opposed to sex, but empirical evidence for the effect of sociocultural vs biological factors is lacking. ${ }^{83}$ Since suboptimal decisions to investigate cancer are likely precipitated by a combination of factors, ${ }^{84}$ future research should examine possible interactions between PCP factors (and patient and health system factors). This is pertinent given the potential for reverse causality among some PCP factors.

\section{Implications for policy and practice}

As the majority of studies in this review were conducted in European countries, our findings have most applicability for countries where PCPs are gatekeepers to specialty services. Our findings have implications for improving the management of symptomatic patients with cancer in general rather than a specific cancer type. This is of clinical import as patients may be at lower risk of an individual cancer but higher risk of an underlying cancer overall. ${ }^{3485}$ In the absence of clinical judgement of symptoms as 'alarm' or 'gut feeling', PCPs may not investigate patients in a timely manner. Novel pathways for non-specific symptoms (eg, SCAN pathway in Oxford ${ }^{86}$ ) that are currently in development in the UK, will be important for circumventing perceived barriers to acting on 'clinical judgement' or 'gut feeling' for symptoms not meeting urgent referral criteria. However, supplementary strategies are needed that support PCPs to recognise and investigate patients with non-alarm and non-specific symptoms that need referral. The challenge for policymakers will be to determine how to enhance the utility of clinical guidelines for investigation of possible cancer by operationalising clinical suspicion/judgement and 'gut feeling', and ensuring recommendations refrain from reinforcing language siloes between patients and PCPs that may create opportunities for suboptimal testing decisions and diagnostic delay.

\section{Author affiliations}

${ }^{1}$ The Primary Care Unit, Department of Public Health and Primary Care, University of Cambridge, Cambridge, UK

${ }^{2}$ College of Medicine and Health, University of Exeter, Exeter, UK

${ }^{3}$ Department of Family Medicine, University of Washington, Seattle, Washington, USA

${ }^{4}$ Centre for Cancer Research and Department of General Practice, University of Melbourne VCCC, Parkville, Victoria, Australia

${ }^{5}$ Wolfson Institute of Population Health, Barts and The London School of Medicine and Dentistry, Queen Mary University of London, London, UK

Twitter Samuel William David Merriel @sammerriel

Contributors VH, MT, JE and FMW designed the study; VH developed and performed the search; $\mathrm{VH}, \mathrm{AY}$ and SWDM screened the search results; $\mathrm{VH}, \mathrm{AY}$ and SA extracted the data; VH drafted the manuscript with input from JU-S and FMW; 
all authors critically assessed and contributed to manuscript revisions. FMW is guarantor of this work

Funding This review is supported by the CanTest Collaborative which is funded by Cancer Research UK (C8640/A23385), where VH is a PhD student, SWDM is a Clinical Research Fellow, FMW is Director, and MT and JE are associate directors. SA is supported by Cancer Research UK grants (C12292/A20861).

Competing interests None declared.

Patient consent for publication Not applicable.

Provenance and peer review Not commissioned; externally peer reviewed.

Data availability statement All data relevant to the study are included in the article or uploaded as online supplemental information. All research data supporting this publication are provided within this article.

Supplemental material This content has been supplied by the author(s). It has not been vetted by BMJ Publishing Group Limited (BMJ) and may not have been peer-reviewed. Any opinions or recommendations discussed are solely those of the author(s) and are not endorsed by BMJ. BMJ disclaims all liability and responsibility arising from any reliance placed on the content. Where the content includes any translated material, BMJ does not warrant the accuracy and reliability of the translations (including but not limited to local regulations, clinical guidelines, terminology, drug names and drug dosages), and is not responsible for any error and/or omissions arising from translation and adaptation or otherwise

Open access This is an open access article distributed in accordance with the Creative Commons Attribution Non Commercial (CC BY-NC 4.0) license, which permits others to distribute, remix, adapt, build upon this work non-commercially, and license their derivative works on different terms, provided the original work is properly cited, appropriate credit is given, any changes made indicated, and the use is non-commercial. See: http://creativecommons.org/licenses/by-nc/4.0/.

\section{ORCID iDs}

Victoria Hardy http://orcid.org/0000-0002-8620-4404

Stephanie Archer http://orcid.org/0000-0003-1349-7178

Samuel William David Merriel http://orcid.org/0000-0003-2919-9087

Matthew Thompson http://orcid.org/0000-0003-0256-8444

Jon Emery http://orcid.org/0000-0002-5274-6336

Juliet Usher-Smith http://orcid.org/0000-0002-8501-2531

Fiona M Walter http://orcid.org/0000-0002-7191-6476

\section{REFERENCES}

1 Singh H, Schiff GD, Graber ML, et al. The global burden of diagnostic errors in primary care. BMJ Qual Saf 2017;26:484-94.

2 Fernholm R, Pukk Härenstam K, Wachtler C, et al. Diagnostic errors reported in primary healthcare and emergency departments: a retrospective and descriptive cohort study of 4830 reported cases of preventable harm in Sweden. Eur J Gen Pract 2019;25:128-35.

3 Aaronson EL, Quinn GR, Wong Cl, et al. Missed diagnosis of cancer in primary care: insights from malpractice claims data. Journal of Healthcare Risk Management 2019;39:19-29.

4 Kostopoulou O, Delaney BC, Munro CW. Diagnostic difficulty and error in primary care--a systematic review. Fam Pract 2008;25:400-13.

5 World Health organization. Diagnostic errors: technical series on safer primary care. Geneva, 2016.

6 Hamilton W. Cancer diagnosis in primary care. Br J Gen Pract 2010;60:121-8.

7 Swann R, McPhail S, Witt J, et al. Diagnosing cancer in primary care: results from the National cancer diagnosis audit. $\mathrm{Br} J$ Gen Pract 2018;68:e63-72.

8 Koo MM, Swann R, McPhail S, et al. The prevalence of chronic conditions in patients diagnosed with one of 29 common and rarer cancers: a cross-sectional study using primary care data. Cancer Epidemiol 2020:69:101845.

9 Koo MM, von Wagner C, Abel GA, et al. The nature and frequency of abdominal symptoms in cancer patients and their associations with time to help-seeking: evidence from a national audit of cancer diagnosis. J Public Health 2018;40:e388-95.

10 Stapley S, Peters TJ, Neal RD, et al. The risk of oesophago-gastric cancer in symptomatic patients in primary care: a large case-control study using electronic records. Br J Cancer 2013;108:25-31.

11 National collaborating centre for cancer. Suspected cancer: recognition and referral NICE guideline. full guideline, 2015. https://www.nice.org.uk/ guidance/ng12/evidence/full-guidelinepdf-2676000277

12 Funston G, Van Melle M, Baun M-LL, et al. Variation in the initial assessment and investigation for ovarian cancer in symptomatic women: a systematic review of international guidelines. BMC Cancer 2019;19:1-13.

13 Neal RD, Din NU, Hamilton W, et al. Comparison of cancer diagnostic intervals before and after implementation of NICE guidelines: analysis of data from the UK general practice research database. Br J Cancer 2014;110:584-92.

14 Jensen $\mathrm{H}$, Tørring ML, Olesen F, et al. Diagnostic intervals before and after implementation of cancer patient pathways - a GP survey and registry based comparison of three cohorts of cancer patients. BMC Cancer 2015;15:1-10.

15 Swann R, Lyratzopoulos G, Rubin G, et al. The frequency, nature and impact of GP-assessed avoidable delays in a population-based cohort of cancer patients. Cancer Epidemiol 2020;64:101617.

16 Mendonca SC, Abel GA, Saunders CL, et al. Pre-referral general practitioner consultations and subsequent experience of cancer care: evidence from the English cancer patient experience survey. Eur $J$ Cancer Care 2016;25:478-90.

17 Black G, Sheringham J, Spencer-Hughes V, et al. Patients' Experiences of Cancer Diagnosis as a Result of an Emergency Presentation: A Qualitative Study. PLoS One 2015;10:e0135027-17.

18 Abel G, Saunders CL, Mendonca SC, et al. Variation and statistical reliability of publicly reported primary care diagnostic activity indicators for cancer: a cross-sectional ecological study of routine data. BMJ Qual Saf 2018;27:21-30.

19 Burton C, O'Neill L, Oliver P. Contribution of primary carer organisation and specialist care provider to variation in GP referrals for suspected cancer: ecological analysis of national data. BMJ Qual Saf 2019;29:274-6.

20 Meechan D, Gildea C, Hollingworth L, et al. Variation in use of the 2-week referral pathway for suspected cancer: a cross-sectional analysis. Br J Gen Pract 2012;62:e590-7.

21 Moher D, Liberati A, Tetzlaff J, et al. Preferred reporting items for systematic reviews and meta-analyses: the PRISMA statement. BMJ 2009;339:b2535-6.

22 King's Fund. An independent audit of the NHS under labour (19972005), 2005. Available: https://www.kingsfund.org.uk/publications/ independent-audit-nhs-under-labour-1997-2005

23 Sheringham J, Sequeira R, Myles J, et al. Variations in GPs' decisions to investigate suspected lung cancer: a factorial experiment using multimedia vignettes. BMJ Qual Saf 2017;26:449-59.

24 Whiting P, Toerien M, de Salis I, et al. A review identifies and classifies reasons for ordering diagnostic tests. J Clin Epidemiol 2007;60:981-9.

25 Kostopoulou O, Sirota M, Round T, et al. The role of physicians' first impressions in the diagnosis of possible cancers without alarm symptoms. Med Decis Making 2017;37:9-16.

26 Wenghofer EF, Williams AP, Klass DJ. Factors affecting physician performance: implications for performance improvement and governance. Healthc Policy 2009;5:141-60.

27 Patient care and the general practitioner. BMJ 1994;309:1144-6.

28 Ouzzani M, Hammady H, Fedorowicz Z, et al. Rayyan - a web and mobile APP for systematic reviews. Syst Rev 2016;5:1-10.

29 McHugh ML. Interrater reliability: the kappa statistic. Biochem Med 2012;22:276-82.

30 Joanna Briggs Institute. Joanna Briggs Institute critical appraisal tools, 2016. Available: https://joannabriggs.org/research/criticalappraisal-tools.html

31 Jones OT, Jurascheck LC, van Melle MA, et al. Dermoscopy for melanoma detection and triage in primary care: a systematic review. BMJ Open 2019;9:e027529.

32 The Joanna Briggs Institute. The Joanna Briggs Institute Reviewers' Manual 2014: the systematic review of economic evaluation evidence since, 2014. Available: https://nursing.Isuhsc.edu/JBI/docs/ ReviewersManuals/Economic.pdf

33 Popay J, Roberts H, Sowden A. Guidance on the conduct of narrative synthesis in systematic reviews. A product from the ESRC methods programme, 2006.

34 Chapman D, Poirier V, Vulkan D, et al. First results from five multidisciplinary diagnostic centre (MDC) projects for non-specific but concerning symptoms, possibly indicative of cancer. Br J Cancer 2020;123:722-9.

35 Ramanathan SA, Baratiny G, Stocks NP, et al. General practitioner referral patterns for women with gynaecological symptoms: a randomised incomplete block study design. Med J Aust 2011;195:602-6.

36 Rogers HL, Dumenci L, Epstein RM, et al. Impact of patient gender and race and physician communication on colorectal cancer 
diagnostic visits in primary care. Journal of Women's Health 2019;28:612-20.

37 Kostopoulou O, Nurek M, Delaney BC. Disentangling the relationship between physician and organizational performance: a signal detection approach. Med Decis Making 2020;40:746-55.

38 Goff BA, Matthews B, Andrilla CHA, et al. How are symptoms of ovarian cancer managed? A study of primary care physicians. Cancer 2011;117:4414-23.

39 Harris M, Brekke M, Dinant G-J, et al. Primary care practitioners' diagnostic action when the patient may have cancer: an exploratory vignette study in 20 European countries. BMJ Open 2020;10:e035678.

40 Ingeman ML, Christensen MB, Bro F, et al. The Danish cancer pathway for patients with serious non-specific symptoms and signs of cancer-a cross-sectional study of patient characteristics and cancer probability. BMC Cancer 2015;15:1-11.

41 Jensen $\mathrm{H}$, Tørring ML, Olesen $\mathrm{F}$, et al. Cancer suspicion in general practice, urgent referral and time to diagnosis: a population-based GP survey and registry study. BMC Cancer 2014;14.

42 Jiwa M, Gordon M, Arnet H, et al. Referring patients to specialists: a structured vignette survey of Australian and British GPs. BMC Fam Pract 2008:9:1-7.

43 John SKP, Jones OM, Horseman N, et al. Inter general practice variability in use of referral guidelines for colorectal cancer. Colorect Dis 2007:9:731-5.

44 Kostopoulou O, Nurek M, Cantarella S, et al. Referral decision making of general practitioners: a signal detection study. Med Decis Making 2019;39:21-31.

45 Pedersen A, Vedsted P. General practitioners' anticipated risk of cancer at referral and their attitude to risk taking and to their role as gatekeeper. J Health Serv Res Policy 2015;20:210-6.

46 Brownell P, Piccolo F, Brims F. Does this lung nodule need urgent review? a discrete choice experiment of Australian general practitioners. BMC Pulm Med 2020;20:1-8.

47 Hjertholm P, Moth G, Ingeman ML, et al. Predictive values of GPs' suspicion of serious disease: a population-based follow-up study. $\mathrm{Br}$ $J$ Gen Pract 2014;64:e346-53.

48 Guldbrandt LM, Rasmussen TR, Rasmussen F, et al. Implementing direct access to low-dose computed tomography in general practice-method, adaption and outcome. PLoS One 2014;9:e112162.

49 Scheel BI, Ingebrigtsen SG, Thorsen T, et al. Cancer suspicion in general practice: the role of symptoms and patient characteristics, and their association with subsequent cancer. Br J Gen Pract 2013;63:e627-35.

50 Friedemann Smith C, Kristensen BM, Andersen RS. GPs' use of gut feelings when assessing cancer risk in primary care: a qualitative study. Br J Gen Pract2020;71:e356-63.

51 Green T, Atkin K, Macleod U. Cancer detection in primary care: insights from general practitioners. Br J Cancer 2015;112 Suppl 1:S41-9.

52 Högberg C, Samuelsson E, Lilja M, et al. Could it be colorectal cancer? General practitioners' use of the faecal occult blood test and decision making - a qualitative study. BMC Fam Pract 2015;16:1-8.

53 Johansen M-L, Holtedahl KA, Rudebeck CE. How does the thought of cancer arise in a general practice consultation? interviews with GPs. Scand J Prim Health Care 2012;30:135-40.

54 Kidney E, Greenfield S, Berkman L, et al. Cancer suspicion in general practice, urgent referral, and time to diagnosis: a populationbased GP survey nested within a feasibility study using information technology to flag-up patients with symptoms of colorectal cancer. BJGP Open 2017;1:bjgpopen17X101109.

55 Wagland R, Brindle L, James E, et al. Facilitating early diagnosis of lung cancer amongst primary care patients: the views of GPs. Eur J Cancer Care 2017;26:e12704-8.

56 Harris M, Frey P, Esteva M. How health system factors influence referral decisions in patients that may have cancer: European symposium report. J Cancer Res Ther 2016;4:7-10.

57 Dodds W, Morgan M, Wolfe C, et al. Implementing the 2-week wait rule for cancer referral in the UK: general practitioners' views and practices. Eur J Cancer Care 2004;13:82-7.

58 Harris M, Thulesius H, Neves AL, et al. How European primary care practitioners think the timeliness of cancer diagnosis can be improved: a thematic analysis. BMJ Open 2019;9:e030169-10.

59 van Erp NF, Helsper CW, Olyhoek SM, et al. Potential for reducing time to referral for colorectal cancer patients in primary care. Ann Fam Med 2019;17:419-27.

60 Baun M-LL, Jensen H, Falborg AZ, et al. Ovarian cancer suspicion, urgent referral and time to diagnosis in Danish general practice: a population-based study. Fam Pract 2019;36:751-7.

61 Guldbrandt LM, Fenger-Grøn M, Rasmussen TR, et al. The role of general practice in routes to diagnosis of lung cancer in Denmark: a population-based study of general practice involvement, diagnostic activity and diagnostic intervals. BMC Health Serv Res 2015;15:21.

62 Walter FM, Mills K, Mendonça SC, et al. Symptoms and patient factors associated with diagnostic intervals for pancreatic cancer (symptom pancreatic study): a prospective cohort study. Lancet Gastroenterol Hepatol 2016;1:298-306.

63 Joyce K, Zermanos T, Badrinath P. Factors associated with variation in emergency diagnoses of cancer at general practice level in England. Eur J Public Health2020;31:1-8.

64 Rose PW, Rubin G, Perera-Salazar R, et al. Explaining variation in cancer survival between 11 jurisdictions in the International cancer benchmarking partnership: a primary care vignette survey. BMJ Open 2015;5:e007212

65 Harris M, Vedsted P, Esteva M, et al. Identifying important health system factors that influence primary care practitioners' referrals for cancer suspicion: a European cross-sectional survey. BMJ Open 2018;8:e022904.

66 Balogh EP, Miller BT, Ball JR. Improving diagnosis in health care, 2016: 1-472.

67 Gillard S, Benson J, Silverman J. Teaching and assessment of explanation and planning in medical schools in the United Kingdom: cross sectional questionnaire survey. Med Teach 2009;31:328-31.

68 Keijzers G, Fatovich DM, Egerton-Warburton D, et al. Deliberate clinical inertia: using meta-cognition to improve decision-making Emerg Med Australas 2018;30:585-90.

69 Meyer AND, Singh H. Calibrating how doctors think and seek information to minimise errors in diagnosis. BMJ Qual Saf 2017;26:436-8

70 Whelehan DF, Conlon KC, Ridgway PF. Medicine and heuristics: cognitive biases and medical decision-making. Ir J Med Sci 2020;189:1477-84.

71 del Campo C, Pauser S, Steiner E, et al. Decision making styles and the use of heuristics in decision making. J Bus Econ 2016;86:389-412.

72 Pedersen AF, Ingeman ML, Vedsted P. Empathy, burn-out and the use of gut feeling: a cross-sectional survey of Danish general practitioners. BMJ Open 2018;8:e020007-8.

73 Kristensen BM, Andersen RS, Nicholson BD, et al. Cultivating doctors' gut feeling: experience, temporality and politics of gut feelings in family medicine. Cult Med Psychiatry 2021. doi:10.1007/ s11013-021-09736-3. [Epub ahead of print: 26 Sep 2021].

74 Smith CF, Drew S, Ziebland S, et al. Understanding the role of GPs gut feelings in diagnosing cancer in primary care: a systematic review and meta-analysis of existing evidence. $\mathrm{Br} J$ Gen Pract 2020;70:e612-21.

75 Winter R, Issa E, Roberts N, et al. Assessing the effect of empathyenhancing interventions in health education and training: a systematic review of randomised controlled trials. BMJ Open 2020;10:1-12.

76 Zwaan L, El-Kareh R, Meyer AND, et al. Advancing diagnostic safety research: results of a systematic research priority setting exercise. $J$ Gen Intern Med 2021;36:2943-51.

77 Din NU, Ukoumunne OC, Rubin G, et al. Age and gender variations in cancer diagnostic intervals in 15 cancers: analysis of data from the UK clinical practice research Datalink. PLoS One 2015;10:e0127717.

78 Williams $\mathrm{P}$, Murchie $\mathrm{P}$, Bond $\mathrm{C}$. Patient and primary care delays in the diagnostic pathway of gynaecological cancers: a systematic review of influencing factors. Br J Gen Pract 2019;69:E106-11.

79 Humphrys E, Walter FM, Rubin G, et al. Patient symptom experience prior to a diagnosis of oesophageal or gastric cancer: a multimethods study. BJGP Open 2020;4:1-15.

80 Bankhead CR, Collins C, Stokes-Lampard H, et al. Identifying symptoms of ovarian cancer: a qualitative and quantitative study. BJOG 2008;115:1008-14.

81 Mills K, Birt L, Emery JD, et al. Understanding symptom appraisal and help-seeking in people with symptoms suggestive of pancreatic cancer: a qualitative study. BMJ Open 2017;7:1-9.

82 Mendonca SC, Abel GA, Gildea C, et al. Associations between general practice characteristics with use of urgent referrals for suspected cancer and endoscopies: a cross-sectional ecological study. Fam Pract 2019;36:573-80.

83 Champagne-Langabeer T, Hedges AL. Physician gender as a source of implicit bias affecting clinical decision-making processes: a scoping review. BMC Med Educ 2021;21:1-9.

84 Graber ML, Franklin N, Gordon R. Diagnostic error in internal medicine. Arch Intern Med 2005;165:1493-9.

85 Herbert A, Rafiq M, Pham TM, et al. Predictive values for different cancers and inflammatory bowel disease of 6 common abdominal symptoms among more than 1.9 million primary care patients in the UK: a cohort study. PLoS Med 2021;18:e1003708. 
86 Nicholson BD, Oke J, Friedemann Smith C, et al. The suspected cancer (scan) pathway: protocol for evaluating a new standard of care for patients with non-specific symptoms of cancer. BMJ Open 2018;8:1-8.

87 Stolper E, van Bokhoven M, Houben P, et al. The diagnostic role of gut feelings in general practice a focus group study of the concept and its determinants. BMC Fam Pract 2009;10:1-9.

88 Pedersen AF, Andersen CM, Ingeman ML, et al. Patient-physician relationship and use of gut feeling in cancer diagnosis in primary care: a cross-sectional survey of patients and their general practitioners. BMJ Open 2019;9:e027288-10.
89 Woolley A, Kostopoulou O. Clinical intuition in family medicine: more than first impressions. Ann Fam Med 2013;11:60-6.

90 Arroll B, Allan GM, Elley CR, et al. Diagnosis in primary care: probabilistic Reasoning. J Prim Health Care 2012;4:166-73.

91 Hillen MA, Gutheil CM, Strout TD, et al. Tolerance of uncertainty: conceptual analysis, integrative model, and implications for healthcare. Soc Sci Med 2017;180:62-75.

92 VanNieuwenborg L, Goossens M, De Lepeleire J, et al. Continuing medical education for general practitioners: a practice format. Postgrad Med J 2016;92:217-22. 